Check for updates

Cite this: RSC Adv., 2020, 10, 2483

Received 7th November 2019

Accepted 4th January 2020

DOI: $10.1039 / \mathrm{c} 9 \mathrm{ra0} 9252 \mathrm{~h}$

rsc.li/rsc-advances

\section{Polyethyleneimine-interlayered silica-core quantum dot-shell nanocomposites for sensitive detection of Salmonella typhimurium via a lateral flow immunoassay $\dagger$}

\author{
Bo Zhang, ${ }^{\text {ad }}$ Xingsheng Yang, ${ }^{\text {bc }}$ Xiaoxian Liu, ${ }^{\text {bc }}$ Juan Li, (DD *a Chongwen Wang (D) *bc \\ and Shengqi Wang*bc
}

Herein, we synthesized high-performance $\mathrm{SiO}_{2}$-core quantum dot (QD)-shell nanocomposites $\left(\mathrm{SiO}_{2} @ \mathrm{CPEI}-\mathrm{QDs}\right)$ using the polyethyleneimine (PEI)-mediated adsorption method. Cationic PEI was used to form a positively charged interlayer on the $\mathrm{SiO}_{2}$ core, which achieved a dense adsorption of carboxylated QDs to form a shell of QDs and maintained a good dispersibility of the nanocomposite. The $\mathrm{SiO}_{2} @ \mathrm{QPEI}-\mathrm{QDs}$ showed excellent stability and high luminescence, and served as high-performance fluorescent labels for the detection of bacteria when used with the lateral flow immunoassay (LFA) technique. An $\mathrm{SiO}_{2} @ \mathrm{QPEI}-\mathrm{QD}$-based LFA strip was successfully applied to rapidly detect Salmonella typhimurium in milk samples with a low limit of $5 \times 10^{2}$ cells per $\mathrm{mL}$.
Salmonella typhimurium (S. typhi) is one of main foodborne pathogens affecting humans and animals worldwide; it causes great damage to public health, high mortality, and high economic loss. ${ }^{1,2}$ Current mature methods for detecting $S$. typhi primarily include the conventional plate culturing, polymerase chain reaction, DNA sequencing, and enzyme-linked immunosorbent assay techniques. ${ }^{2-5}$ However, these methods have several inherent shortcomings, such as requiring tedious procedures and long testing times, multistep sample pretreatment, expensive equipment, and skilled personnel, for the rapid detection of bacteria. ${ }^{6}$ Thus, a sensitive and convenient technique for rapid detection of pathogenic bacteria must be developed.

The lateral flow immunoassay (LFA) technique has become one of the most efficient point-of-care testing tools because of its distinct advantages of simple operation, rapid analysis, low cost, and flexibility for different tested substances. ${ }^{7-9}$ Quantum dots (QDs) as novel fluorescent labels are used in LFAs to improve detection sensitivity and quantitative ability owing to their superior optical properties including photostability, strong luminescence, and narrow fluorescence emission

${ }^{a}$ School of Public Health, Jilin University, Changchun 130021, PR China. E-mail: li_juan@jlu.edu.cn

${ }^{b}$ College of Life Sciences, Anhui Agricultural University, Hefei 230036, PR China. E-mail: wangchongwen1987@126.com; sqwang@bmi.ac.cn

${ }^{c}$ Beijing Institute of Radiation Medicine, Beijing 100850, PR China

${ }^{d}$ Department of Pharmacy, Peking Union Medical College Hospital, Chinese Academy Medical Sciences \& Peking Union Medical College, Beijing 100730, PR China

$\dagger$ Electronic supplementary information (ESI) available. See DOI: 10.1039/c9ra09252h spectral peaks. ${ }^{10-12}$ However, QDs are also subject to several key problems during bioanalysis, such as their tendency to easily aggregate, instability in complex solutions, and lack of biocompatibility. ${ }^{13}$ These problems can be overcome by combining QDs and other support materials, such as latex beads, ${ }^{14} \mathrm{SiO}_{2},{ }^{15}$ polymeric microbeads, ${ }^{16}$ and $\mathrm{Fe}_{3} \mathrm{O}_{4},{ }^{17}$ into one micro- or nanosphere. However, most of the reported QD composites have dimensions generally greater than $300 \mathrm{~nm}$ and the methods used to synthesize them are complex, which have greatly restricted their application in LFA systems for the detection of bacteria.

Here, we report a facile polyethyleneimine (PEI)-mediated adsorption strategy to fabricate PEI-interlayered $\mathrm{SiO}_{2}$-core QD-shell nanomaterials ( $\mathrm{SiO}_{2} @$ PEI-QDs) displaying controllable dimensions, monodispersity, and excellent fluorescence. PEI was employed to form an electropositive thin interlayer on the surface of $\mathrm{SiO}_{2}$ NPs not only to achieve a dense adsorption of CdSe/ZnS-MPA QDs as QD shells but also to maintain the stability of the nanostructure in the solution. Our results revealed the performance and stability of $\mathrm{SiO}_{2} @ P E I-Q D s$ and demonstrated that the proposed nanocomposites can act as advanced fluorescent nanotags for QD-based LFA strips. The sensitivity of an $\mathrm{SiO}_{2} @ P E I-Q D-b a s e d$ LFA strip for the detection of $S$. typhi was measured to be as low as $5 \times 10^{2}$ cells per $\mathrm{mL}$. To the best of our knowledge, this work was the first to use highperformance $\mathrm{SiO}_{2}$-QD nanocomposites as fluorescent labels for LFA-based detection of bacteria.

The fabricated $\mathrm{SiO}_{2} @ P E I-Q D s$ were composed of three parts: $\mathrm{SiO}_{2}$ NPs with dimensions of $150 \mathrm{~nm}$ as a hydrophilic support core to provide good dispersity, a thin layer of PEI as an 
electropositive linker, and dense carboxylated QDs forming a shell of QDs to generate high luminescence and surface sites for antibody conjugation. Herein, we chose $\mathrm{SiO}_{2} \mathrm{NPs}$ with dimensions of $150 \mathrm{~nm}$ as the core material because of their uniform size and homogeneous nanostructures, easy preparation, and high stability in complex solutions. CdSe/ZnS-MPA QDs were chosen to form the outer shell of QDs because of the excellent fluorescence properties and numerous surface carboxyl groups of the MPA-modified shell for subsequent antibody coupling. ${ }^{18}$

High-performance $\mathrm{SiO}_{2} @ P E I-Q D s$ with a typical core-shell nanostructure were fabricated in three steps, as shown in Scheme 1a. First, monodisperse $\mathrm{SiO}_{2}$ NPs were synthesized according to a modified Stöber method as the stable core. Second, PEI-coated $\mathrm{SiO}_{2}$ NPs ( $\mathrm{SiO}_{2} @ \mathrm{PEI}$ ) were prepared by dispersing $10 \mathrm{mg}$ of $\mathrm{SiO}_{2} \mathrm{NPs}$ in an aqueous PEI solution $(0.5 \%$, $\mathrm{v} / \mathrm{v}$ ) under sonication for $30 \mathrm{~min}$, during which the cationic polymer PEI quickly self-assembled on the negatively charged $\mathrm{SiO}_{2}$ surface. ${ }^{19-21} \mathrm{SiO}_{2} @$ PEI NPs were collected by carrying out centrifugation, and then dispersed in $5 \mathrm{~mL}$ of deionized water. Finally, $1 \mathrm{~mL}$ of the as-prepared $\mathrm{SiO}_{2} @ P E I$ NPs was added to $100 \mathrm{~mL}$ of a carboxyl-functionalized CdSe/ZnS QD solution (0.1 $\mathrm{nM}$ ) and sonicated for $1 \mathrm{~h}$ to form $\mathrm{SiO}_{2} @ P E I-Q D s$ via electrostatic interaction. The resulting $\mathrm{SiO}_{2} @$ @PI-QDs were collected by carrying out centrifugation, and then stored in $10 \mathrm{~mL}$ of deionized water for further use.

Transmission electron microscopy (TEM) analysis was performed to verify the morphology of the as-synthesized nanocomposites. As shown in Fig. $\mathrm{S} 1 \mathrm{a}, \dagger$ the as-prepared $\mathrm{SiO}_{2} \mathrm{NPs}$ displayed uniforms dimensions of $150 \mathrm{~nm}$ and good dispersibility. The TEM image acquired of commercial CdSe/ZnSMPA QDs is shown in Fig. S1b.† The average particle dimension of the CdSe/ZnS-MPA QDs was approximately $12 \mathrm{~nm}$. After coating $\mathrm{SiO}_{2}$ with PEI by carrying out sonication, the resultant $\mathrm{SiO}_{2}$ @PEI NPs still exhibited good dispersity (Fig. 1a). The acquired high-resolution TEM (HRTEM) image of one of these NPs clearly showed that the thickness of the PEI layer was about $8 \mathrm{~nm}$ (Fig. 1c). Fig. 1b and d show the acquired low- and high-magnification TEM images of the fabricated $\mathrm{SiO}_{2}$ @PEIQDs. Dense CdSe/ZnS QDs were decorated uniformly on the surfaces of the $\mathrm{SiO}_{2} @$ PEI NPs. Moreover, the average diameter of the nanocomposites increased from $150 \mathrm{~nm}$ to $182 \mathrm{~nm}$ after the QD-shell formation. The energy dispersive spectroscopy (EDS) mapping technique was used to confirm the elemental composition of the QD-coated $\mathrm{SiO}_{2}$ nanocomposites. As shown in Fig. 1e, high densities of Cd (green), Se (purple), Zn (red), and $S$ (yellow) were found to surround the Si core (blue). This finding indicated the typical core-shell nanostructure of $\mathrm{SiO}_{2} @ P E I-$ QDs. All of the TEM and EDS results confirmed the successful preparation of the $\mathrm{SiO}_{2} @$ @EI-QDs. Note that the branched PEI effectively attached to the surfaces of the $\mathrm{SiO}_{2}$ NPs in the aqueous solution and easily realized the full surface amino modification of $\mathrm{SiO}_{2}$ NPs, which was the key to achieving a dense adsorption of CdSe/ZnS-MPA QDs as uniform shells of QDs. Though other cationic polymers such as poly(diallyl dimethylammonium chloride) can also self-assemble on the surfaces of $\mathrm{SiO}_{2}$ NPs to adsorb QDs, the $\mathrm{pH}$ value of the reaction solution in these cases needed to be precisely controlled. ${ }^{15}$

As shown in Fig. 2a and b, the zeta potential of the $\mathrm{SiO}_{2} @ \mathrm{PEI}-$ QDs clearly changed during the course of their synthesis. The zeta potential dramatically increased from $-44.7 \mathrm{mV}$ for the $\mathrm{SiO}_{2} \mathrm{NPs}$ to $+52.5 \mathrm{mV}$ for $\mathrm{SiO}_{2} @ \mathrm{PEI}$, i.e., after the coating of the positively charged PEI layer; it then decreased to $+27.8 \mathrm{mV}$ for the $\mathrm{SiO}_{2} @ \mathrm{PEI}-\mathrm{QDs}$, due to the numerous adsorbed negatively

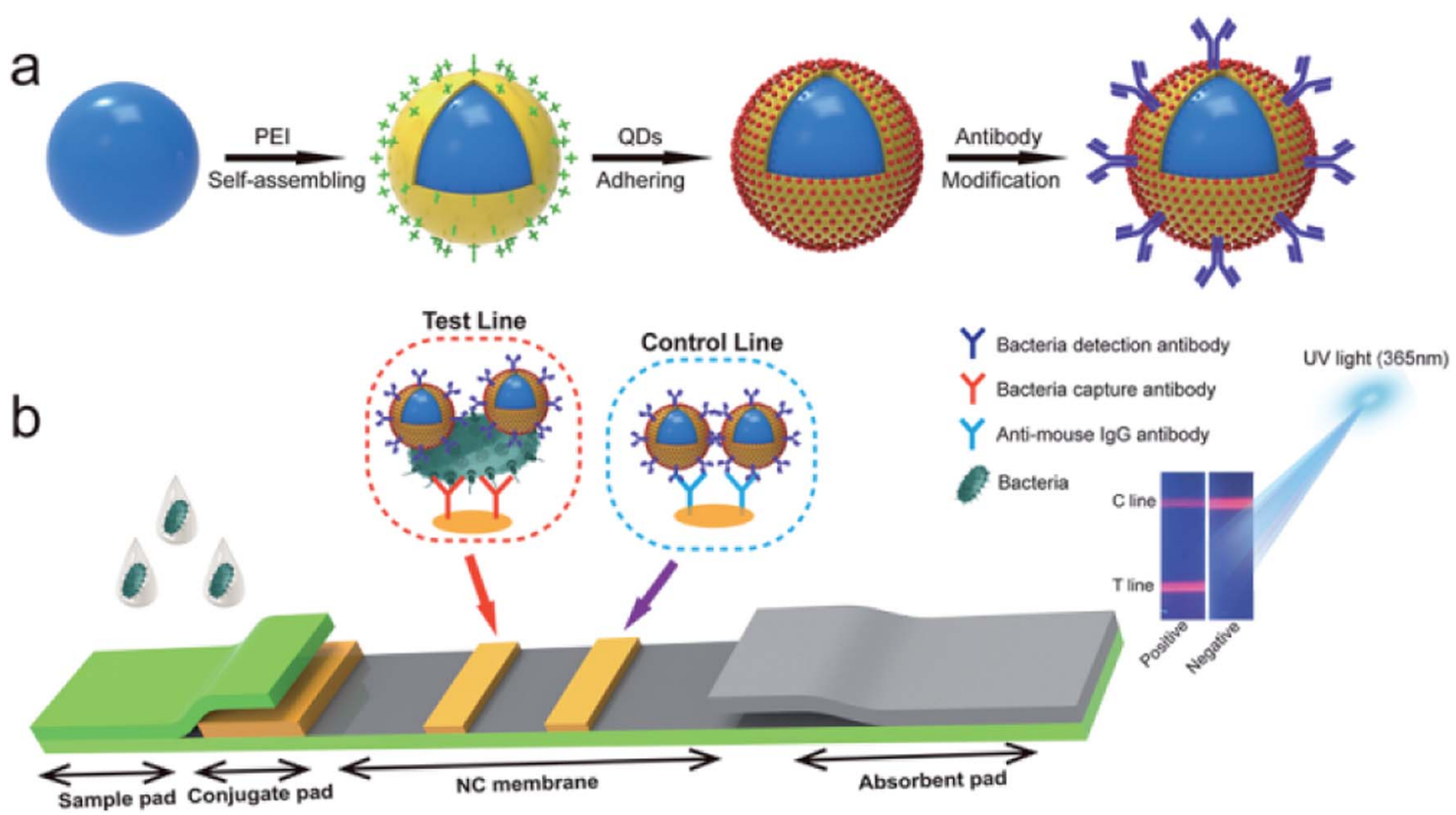

Scheme 1 (a) Schematic of the synthesis of antibody-modified $\mathrm{SiO}_{2}$ (aPEI-QDs. (b) Schematic of the quantitative detection of S. typhi using an $\mathrm{SiO}_{2} @ \mathrm{PEI}-\mathrm{QD}$-based fluorescent LFA strip. 

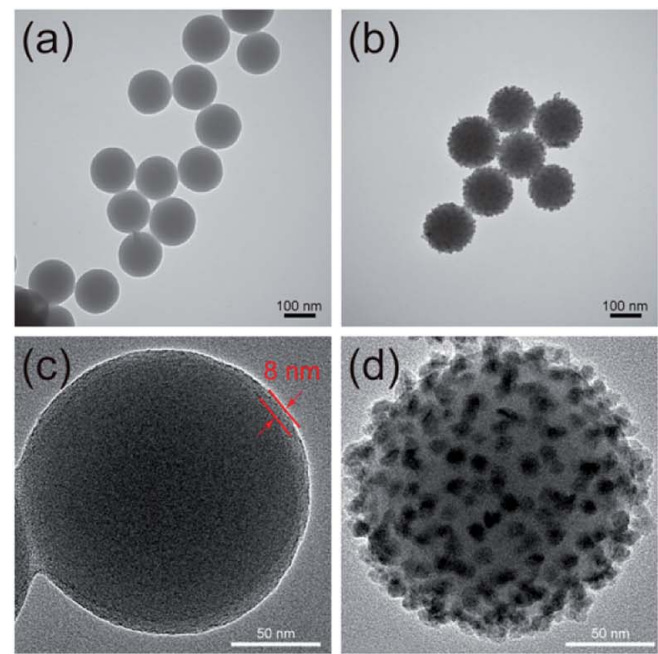

(e)
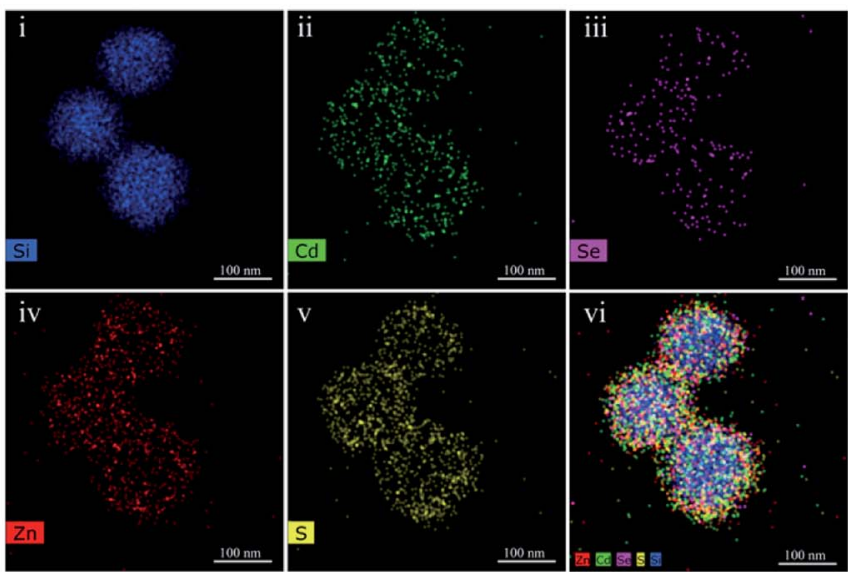

Fig. 1 Characterizations of the morphologies and elemental compositions of the synthesized $\mathrm{SiO}_{2}(\mathrm{aPEI}-\mathrm{QDs}$. (a and b) TEM images of (a) $\mathrm{SiO}_{2} \mathrm{aPEI} N \mathrm{NP}$ and (b) $\mathrm{SiO}_{2} \mathrm{aPEI}-\mathrm{QDs}$. (c and d) Magnified TEM images of (c) a single $\mathrm{SiO}_{2} \mathrm{aPEI} \mathrm{NP}$ and (d) a single $\mathrm{SiO}_{2} @ \mathrm{PPEI}-\mathrm{QDs} \mathrm{NP}$. (e) Elemental mapping images of $\mathrm{SiO}_{2}$ (CPEI-QDs.

charged QDs. These results confirmed that the formation of the $\mathrm{SiO}_{2} @$ PEI-QDs was based on PEI-mediated electrostatic adsorption. We next studied the fluorescence properties of the $\mathrm{SiO}_{2} @$ PEI-QDs. Fig. 2c(i) and (ii) show $\mathrm{SiO}_{2}, \mathrm{SiO}_{2} @ P E I$, and $\mathrm{SiO}_{2} @$ PEI-QD suspensions under visible and $365 \mathrm{~nm}$ wavelength ultraviolet (UV) light, respectively. A bright-red optical emission was observed for $\mathrm{SiO}_{2} @ P E I-Q D s$ excited with a UV light source, whereas $\mathrm{SiO}_{2}$ and $\mathrm{SiO}_{2} @ P E I$ groups showed no fluorescence signal. Fig. 2d shows the corresponding fluorescence emission spectra of the as-synthesized nanocomposites; these spectra revealed the superior fluorescence performance of the $\mathrm{SiO}_{2} @ P E I-Q D s$. Moreover, the fluorescence intensity of $\mathrm{SiO}_{2} @ P E I-Q D s$ remained unchanged for three months when stored in ethanol (Fig. S2 $\dagger$ ), demonstrating their high fluorescence stability. The outstanding fluorescence properties and stability enabled $\mathrm{SiO}_{2} @ P E I-Q D s$ to, as described next, act as high-performance fluorescent labels for LFA stripbased detection of bacteria.

Scheme $1 \mathrm{~b}$ illustrates the experimental principle of the use of $\mathrm{SiO}_{2} @$ PEI-QD-based fluorescence LFA to detect bacteria. The (a)
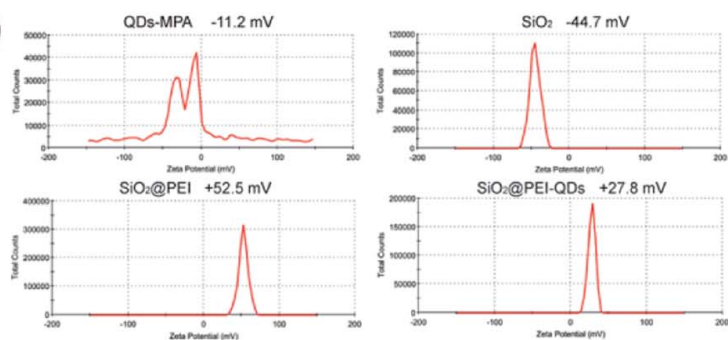

(b)

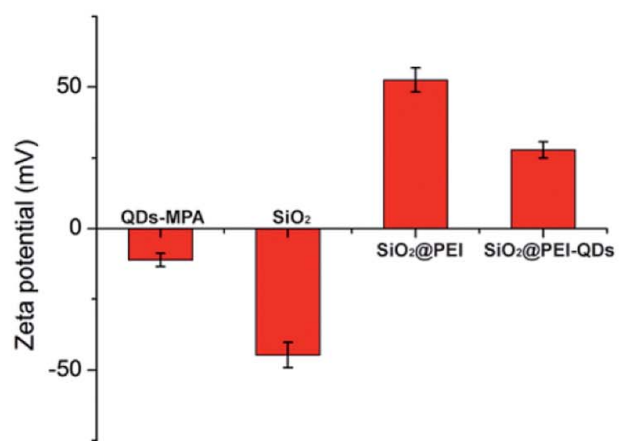

(c)
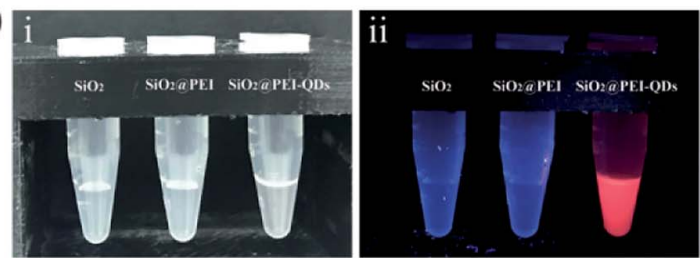

(d)

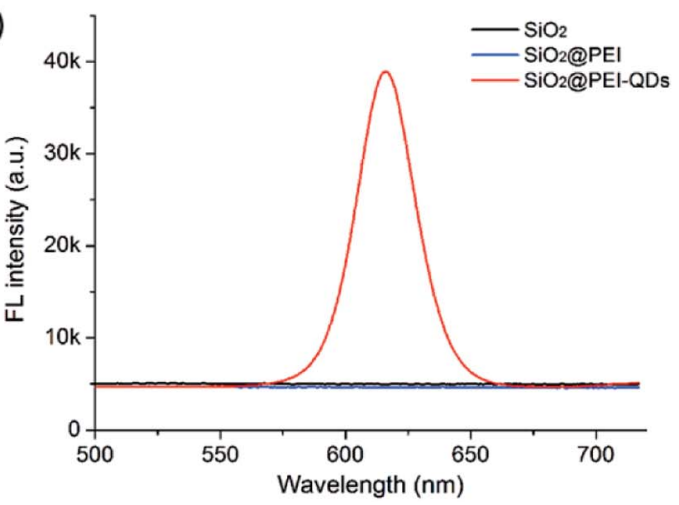

Fig. 2 Characterizations of the electric charge and fluorescence properties of the synthesized $\mathrm{SiO}_{2}(\mathrm{aPEI}-\mathrm{QDs}$. (a) Zeta potentials and (b) statistical analysis of the zeta potentials of the as-obtained nanocomposites at different stages of their synthesis. (c) Photographs of $\mathrm{SiO}_{2}$, $\mathrm{SiO}_{2}\left(\mathrm{aPEl}\right.$, and $\mathrm{SiO}_{2}(\mathrm{aPEI}-\mathrm{QDs}$ suspensions under visible (i) and UV light (ii). (d) Fluorescence emission spectra of these particles in deionized water. 
strip system consisted of four independent parts: a sample pad, conjugate pad, nitrocellulose (NC) membrane, and absorbent pad. Anti-S. typhi monoclonal antibody and goat anti-mouse IgG antibody were simultaneously dispensed into the NC membrane to form test and control lines, respectively. When the strip was inserted into the sample solution, the solution moved toward the absorbent pad through capillary force. In the presence of $S$. typhi, $\mathrm{SiO}_{2} @$ PEI-QDs modified with the $S$. typhi monoclonal antibody recognized and bound to the target bacteria and were finally captured on the test zone by forming $\mathrm{SiO}_{2} @$ @PI-QD-S. typhi-antibody sandwich immune complexes. Superfluous immune $\mathrm{SiO}_{2} @$ PEI-QDs continued to move forward and were immobilized on the control line of the strip. Finally, bacteria were quantitatively analyzed by recording the fluorescence intensity of the test line with a fluorescence reader (with 365 nm-wavelength excitation).

Immuno-SiO ${ }_{2} @ P E I-Q D s$ were prepared by labeling the surface carboxyl groups of $\mathrm{SiO}_{2} @ P E I-Q D s$ directly with $S$. typhi antibodies via carbodiimine chemistry. The zeta potential value decreased from $+27.8 \mathrm{mV}$ for the $\mathrm{SiO}_{2} @$ PEI-QDs to $+12.7 \mathrm{mV}$ for the immuno-SiO ${ }_{2} @ P E I-Q D s$, suggesting the successful attachment of antibody molecules onto the surfaces of the $\mathrm{SiO}_{2} @ \mathrm{PEI}-$


showed that they remained monodispersed after the antibody
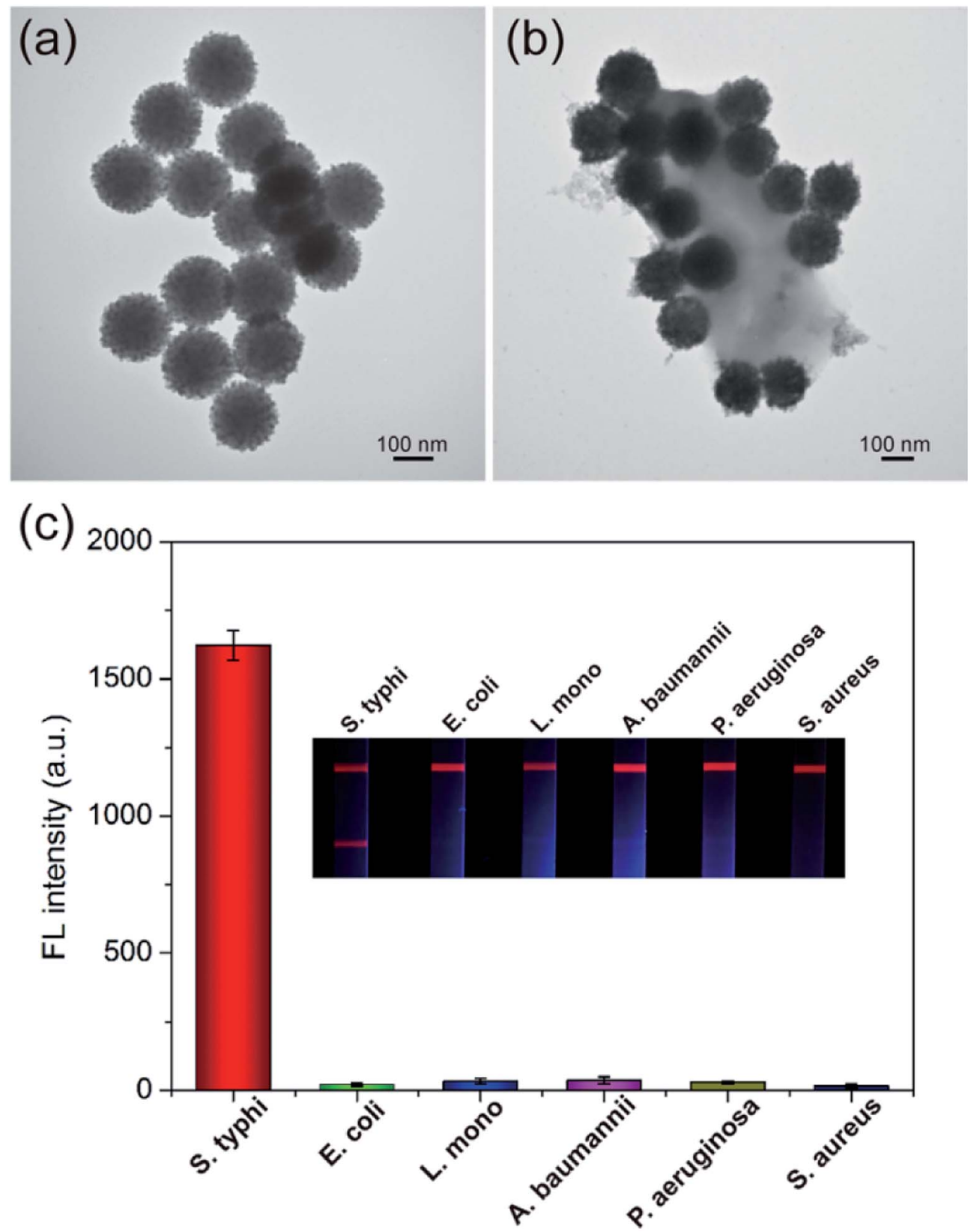

Fig. 3 (a and b) TEM images of (a) immuno-SiO ${ }_{2}$ aPEI-QDs and (b) an immunocomplex of $\mathrm{SiO}_{2} @$ aPEI-QDs and S. typhi. (c) Specificity of the $\mathrm{SiO}_{2} @ \mathrm{CPEI}-\mathrm{QD}$-based fluorescent LFA strip. The inset shows photographs of the test strips in the presence of $\mathrm{S}$. typhi and five interfering bacteria each at a concentration of $10^{5}$ cells per $\mathrm{mL}$. The error bars represent the standard deviations from three repeats of the experiment. 
modification (Fig. 3a). As shown in Fig. 3b, the antibodyconjugated $\mathrm{SiO}_{2} @$ @EI-QDs were directly observed using TEM to effectively bind an $S$. typhi. target.

After preparation of immuno-SiO ${ }_{2}$ @PEI-QDs, the important conditions of the LFA detection system were optimized. The running buffer of the LFA strip was first evaluated because of its direct effect on the flow rate of $\mathrm{SiO}_{2} @ P E I-Q D$ nanotags and the immune binding efficiency of the test line. ${ }^{22,23}$ As shown in Fig. S4a and $b, \uparrow$ the PBST buffer (10 mM, pH 7.4, 1\% Tween 20) ensured a smooth delivery of $\mathrm{SiO}_{2} @ P E I-Q D-b a c t e r i a$ complexes along the strip and achieved the highest signal-to-noise ratio (SNR) on the test line. We then optimized the concentration of the coated antibodies on the test zone. The highest SNR was achieved when the concentration of the $S$. typhi antibody was $0.6 \mathrm{mg} \mathrm{mL} \mathrm{mL}^{-1}$ (Fig. S4c $\dagger$ ). The selectivity and specificity of the LFA system greatly depended on the specific detection antibody applied to the $\mathrm{SiO}_{2} @$ @EI-QDs. The performance of the $S$. typhi monoclonal antibody was then tested. A total of $10^{5}$ cells per $\mathrm{mL}$ of $S$. typhi and five other common pathogenic bacteria including Escherichia coli (E. coli), Listeria monocytogenes (L. mono), Acinetobacter baumannii (A. baumannii), Pseudomonas aeruginosa ( $P$. aeruginosa), and Staphylococcus aureus (S. aureus) were used to test the specificity of the proposed assay. As shown in the inset of Fig. 3c, only $S$. typhi exhibited an evident fluorescence signal on the test line, whereas all nontarget bacteria groups showed no fluorescence signal on the same area. Moreover, bright fluorescence control lines appeared on all of the strips, indicating that the LFA strips were working correctly. The corresponding fluorescence intensities of the test lines were recorded. The results indicated the good selectivity of the $\mathrm{SiO}_{2} @ P E I-Q D-b a s e d$ strip for the detection of $S$. typhi (Fig. 3c). Moreover, the stability and anti-interference ability of the $\mathrm{SiO}_{2} @ P E I-Q D-b a s e d$ strip was evaluated by setting out to detect $S$. typhi in untreated milk and tap water samples. As shown in Fig. S5, $\dagger$ the milk and tap water groups as well as PBS group generated strong fluorescence signals, indicating that the proposed strip can work well in real food samples.

We evaluated the detection sensitivity of the proposed assay in milk samples under the optimized conditions. Fig. 4a(i) and (ii) show photographs and fluorescence pictures of the $\mathrm{SiO}_{2} @ P E I-Q D-$ based strip used to test milk samples spiked with various concentrations of $S$. typhi $\left(10^{7}\right.$ cells per $\mathrm{mL}$ to 0 cells per $\left.\mathrm{mL}\right)$. Under 365 $\mathrm{nm}$-wavelength UV excitation, the red fluorescence band of the test line became darker with decreasing concentration of $S$. typhi in the milk samples. No evident fluorescence signal was observed for the blank group. The visualization limit of the fluorescence signal of the $\mathrm{SiO}_{2} @$ @PE-QD-based strip for $S$. typhi was $10^{3}$ cells per $\mathrm{mL}$. The corresponding test line fluorescence intensities were recorded using a fluorescent strip reader. The fluorescence results were analyzed by plotting the corresponding fluorescence intensities of the test lines as a function of $S$. typhi concentration to produce a calibration curve (Fig. 4b). A linear relationship was observed here within the range $1 \times 10^{4}$ to $5 \times 10^{2}$ cells per $\mathrm{mL}$ for $S$. typhi (inset of Fig. $4 \mathrm{~b}$ ). The limit of detection (LOD) of the $\mathrm{SiO}_{2} @ P E I-Q D-b a s e d$ strip was estimated to be $5 \times 10^{2}$ cells per $\mathrm{mL}$, with the LOD defined as the $3: 1$ threshold ratio with respect to the blank signal. $^{24-26}$ These results revealed that the proposed LFA strip with
$\mathrm{SiO}_{2} @$ PEI-QDs as the fluorescence label displayed high sensitivity and a wide dynamic range for $S$. typhi detection. For comparison, the $S$. typhi concentrations in test milk samples were also confirmed using the traditional plate counting method. As displayed in Fig. S6, $\uparrow$ the number of bacterial colonies grown on the plates was consistent with the LFA-strip detection results, indicating the good accuracy of the strip based on $\mathrm{SiO}_{2} @ P E I-Q D s$. Moreover, the proposed $\mathrm{SiO}_{2} @ P E I-Q D-b a s e d$ strip enabled a rapid detection of $S$. typhi, specifically within 15 minutes, whereas the plate counting method generally requires $8-24 h^{27,28}$ The reproducibility of the $\mathrm{SiO}_{2} @$ @PEI-QD-based LFA strip was also investigated by testing milk samples containing various concentrations of $S$. typhi. Five independent tests were conducted to measure $S$. typhi samples at concentrations of $10^{5}$ and $10^{3}$ cells per mL. As shown in Fig. S7, $\uparrow$ the proposed assay exhibited high repeatability and reliability.

To directly compare the detectability of the proposed $\mathrm{SiO}_{2} @$ @EI-QDs strip with that of a common fluorescent LFA strip, we prepared a QD nanocomposite-based LFA strip by using the same $S$. typhi detection antibody but replaced $\mathrm{SiO}_{2} @ P E I-Q D s$ with commercial QD nanocomposites. As shown in Fig. S8a, $\dagger$ a TEM image acquired of the commercial QD nanocomposites showed them also containing dozens of QDs per particle, but with dispersibility and uniformity levels inferior to those of $\mathrm{SiO}_{2} @$ PEI-QDs. As shown in Fig. S8b, $\uparrow$ the red fluorescence test lines of the QD nanocomposite-based strip for $S$. typh $i$ were observed with the naked eye at a concentration of 1 $\times 10^{4}$ cells per $\mathrm{mL}$. The corresponding calibration curve was

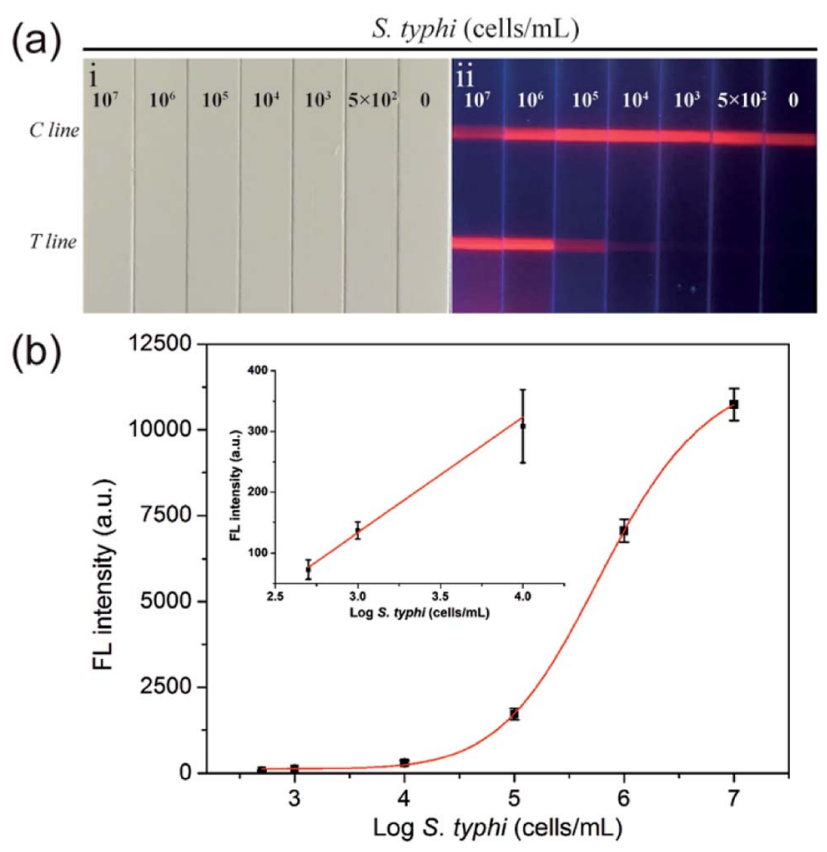

Fig. 4 (a) Photographs (i) and fluorescence pictures (ii) of the $\mathrm{SiO}_{2}(\mathrm{aPEI}-\mathrm{QD}$-based fluorescent LFA strip used for detecting S. typhi. (b) Corresponding test line intensities and calibration curve as a sigmoidal function of the concentration of $S$. typhi within the range $10^{7}$ to 0 cells per $\mathrm{mL}$. The inset shows the linear relationship of the data in the low-concentration range. 
Table 1 Overall performance of the $\mathrm{SiO}_{2}$ ( $\mathrm{PEI}-\mathrm{QD}$-based fluorescent LFA strip compared with other respiratory virus detection techniques

\begin{tabular}{lllll}
\hline Detection method & Bacteria & Detection limit (cells per mL) & Sample & Reference \\
\hline Colorimetric LFA & Salmonella & $10^{3}$ & Milk & Hwang et al. 2016 (ref. 29) \\
Colorimetric LFA & B. cereus & $10^{4}$ & PBS & Kong et al. 2017 (ref. 30 ) \\
Up-converting phosphor LFA & S. typhi & $10^{4}$ & Various foods & Zhao et al. 2017 (ref. 31 ) \\
Colorimetric LFA & E. coli O157 & $4.5 \times 10^{3}$ & Milk & Zhu et al. 2018 (ref. 32) \\
Fluorescent LFA & E. coli O157 & $3 \times 10^{3}$ & Beef, milk Li et al. 2019 (ref. 33) \\
Fluorescent-magnetic LFA & S. typhi & $3.75 \times 10^{3}$ & Milk, blood & Hu et al. 2019 (ref. 34) \\
Fluorescent LFA & S. typhi & $5 \times 10^{2}$ & Milk & This work
\end{tabular}

constructed, as shown in Fig. S8c. $\dagger$ By comparison, the LOD of the $\mathrm{SiO}_{2} @$ PEI-QDs strip was 20 times lower than that of the commercial QD nanocomposite-based LFA strip for $S$. typhi detection. The excellent performance of the assay for the detection of bacteria can be attributed to the advanced $\mathrm{SiO}_{2} @$ PEI-QDs used in the LFA system. These nanocomposites showed numerous shells of QDs, monodispersity, good stability, and a highly reproducible structure. In contrast to other recently reported LFA methods used for detecting bacteria, the proposed $\mathrm{SiO}_{2}$ @PEI-QDs strip showed better sensitivity (Table 1). Moreover, the $\mathrm{SiO}_{2} @$ PEI-QD-based LFA strip can be easily applied for rapid detection of other pathogenic microorganisms by using specific monoclonal antibodies.

In summary, a novel type of $\mathrm{SiO}_{2}$-core QD-shell nanomaterial was fabricated and utilized to prepare bright fluorescent nanotags for LFA strips. By using PEI as the interlayer, numerous carboxyl-functionalized CdSe/ZnS QDs were rapidly and firmly self-assembled on the surfaces of $\mathrm{SiO}_{2} \mathrm{NPs}$, forming a stable nanocomposite with good dispersity, a highly reproducible structure, and high luminescence. Based on the quantitative analysis of $S$. typh $i$ with a detection limit of as low as $5 \times$ $10^{2}$ cells per $\mathrm{mL}$, our results further demonstrated that these $\mathrm{SiO}_{2} @ P E I-Q D s$ can be used as high-performance fluorescent labels for LFA-based detection of bacteria. We believe that the proposed $\mathrm{SiO}_{2} @ P E I-Q D-b a s e d$ LFA strip has great potential for rapid and sensitive detection of pathogens in real samples.

\section{Conflicts of interest}

The authors declare no conflict of interest.

\section{Acknowledgements}

This study was supported by the National S\&T Major Project for Infectious Diseases Control (Grant no. 2018ZX10712001-010, 2018ZX10101003-001), the National Natural Science Foundation of China (Grant no. 81830101), and the Natural Science Foundation of Anhui Province (Grant no. 1908085QB85).

\section{References}

1 G. Li, Y. Huang, M. Duan, K. Xing, X. You, H. Zhou, Y. Liu, C. Liu, D. Liu and W. Lai, Sens. Actuators, B, 2019, 282, 317-321.
2 H. Zhang, L. Xue, F. Huang, S. Wang, L. Wang, N. Liu and J. Lin, Biosens. Bioelectron., 2019, 127, 142-149.

3 X. Xu, X. Ma, H. Wang and Z. Wang, Mikrochim. Acta, 2018, $185,325$.

4 N. Duan, S. Wu, C. Zhu, X. Ma, Z. Wang, Y. Yu and Y. Jiang, Anal. Chim. Acta, 2012, 723, 1-6.

5 J. Chen, S. M. Andler, J. M. Goddard, S. R. Nugen and V. M. Rotello, Chem. Soc. Rev., 2017, 46, 1272-1283.

6 A. Ahmed, J. V. Rushworth, N. A. Hirst and P. A. Millner, Clin. Microbiol. Rev., 2014, 27, 631-646.

7 W. Ren, D. R. Ballou, R. FitzGerald and J. Irudayaraj, Biosens. Bioelectron., 2019, 126, 324-331.

8 D. Zhang, L. Huang, B. Liu, H. Ni, L. Sun, E. Su, H. Chen, Z. Gu and X. Zhao, Biosens. Bioelectron., 2018, 106, 204-211. 9 C. Wang, C. Wang, X. Wang, K. Wang, Y. Zhu, Z. Rong, W. Wang, R. Xiao and S. Wang, ACS Appl. Mater. Interfaces, 2019, 11, 19495-19505.

10 P. Y. You, F. C. Li, M. H. Liu and Y. H. Chan, ACS Appl. Mater. Interfaces, 2019, 11, 9841-9849.

11 M. Xiao, L. Huang, X. Dong, K. Xie, H. Shen, C. Huang, W. Xiao, M. Jin and Y. Tang, Analyst, 2019, 144, 2594-2603.

12 C. Wang, R. Xiao, S. Wang, X. Yang, Z. Bai, X. Li, Z. Rong, B. Shen and S. Wang, Biosens. Bioelectron., 2019, 146, 111754.

13 J. Li, Y. Lv, N. Li, R. Wu, M. Xing, H. Shen, L. S. Li and X. Chen, Chem. Eng. J., 2019, 361, 499-507.

14 S. J. Yeo, H. Kang, T. D. Dao, B. T. Cuc, A. T. V. Nguyen, T. T. T. Tien, N. L. K. Hang, H. V. M. Phuong, L. T. Thanh, L. Q. Mai, Y. Rah, K. Yu, H. J. Shin, C. K. Chong, H. S. Choi and H. Park, Theranostics, 2018, 8, 6132-6148.

15 Y. Zhao, C. Zhou, R. Wu, L. Li, H. Shen and L. S. Li, RSC Adv., 2015, 5, 5988-5995.

16 R. S. Bilan, V. A. Krivenkov, M. A. Berestovoy, A. E. Efimov, I. I. Agapov, P. S. Samokhvalov, I. Nabiev and A. Sukhanova, Chemphyschem, 2017, 18, 970-979.

17 L. Guo, Y. Shao, H. Duan, W. Ma, Y. Leng, X. Huang and Y. Xiong, Anal. Chem., 2019, 91, 4727-4734.

18 Z. Rong, Z. Bai, J. Li, H. Tang, T. Shen, Q. Wang, C. Wang, R. Xiao and S. Wang, Biosens. Bioelectron., 2019, 145, 111719. 19 C. Wang, P. Li, J. Wang, Z. Rong, Y. Pang, J. Xu, P. Dong, R. Xiao and S. Wang, Nanoscale, 2015, 7, 18694-18707.

20 K. Wang, Y. Wang, C. Wang, X. Jia, J. Li, R. Xiao and S. Wang, RSC Adv., 2018, 8, 30825-30831.

21 C. Wang, M. Li, Q. Li, K. Zhang, C. Wang, R. Xiao and S. Wang, RSC Adv., 2017, 7, 13138-13148. 
22 J. Hwang, S. Lee and J. Choo, Nanoscale, 2016, 8, 1141811425.

23 L. W. Yap, H. Chen, Y. Gao, K. Petkovic, Y. Liang, K. J. Si, H. Wang, Z. Tang, Y. Zhu and W. Cheng, Nanoscale, 2017, 9, $7822-7829$.

24 X. Wang, N. Choi, Z. Cheng, J. Ko, L. Chen and J. Choo, Anal. Chem., 2017, 89, 1163-1169.

25 X. Jia, C. Wang, Z. Rong, J. Li, K. Wang, Z. Qie, R. Xiao and S. Wang, RSC Adv., 2018, 8, 21243-21251.

26 Y. Pang, C. Wang, L. Lu, C. Wang, Z. Sun and R. Xiao, Biosens. Bioelectron., 2019, 130, 204-213.

27 Y. Liu, H. Zhou, Z. Hu, G. Yu, D. Yang and J. Zhao, Biosens. Bioelectron., 2017, 94, 131-140.
28 C. Zhang, C. Wang, R. Xiao, L. Tang, J. Huang, D. Wu, S. Liu, Y. Wang, D. Zhang, S. Wang and X. Chen, J. Mater. Chem. B, 2018, 6, 3751-3761.

29 J. Hwang, D. Kwon, S. Lee and S. Jeon, RSC Adv., 2016, 6, 48445-48448.

30 M. Kong, J. H. Shin, S. Heu, J. K. Park and S. Ryu, Biosens. Bioelectron., 2017, 96, 173-177.

31 Y. Zhao, H. Wang, P. Zhang, C. Sun, X. Wang, X. Wang, R. Yang, C. Wang and L. Zhou, Sci. Rep., 2016, 6, 21342.

32 C. Zhu, G. Zhao and W. Dou, Anal. Chim. Acta, 2018, 1038, 97-104.

33 Q. Li, Y. Yang, F. Hu, Y. Cai, X. Liu and X. He, Anal. Biochem., 2019, 564-565, 32-39.

34 J. Hu, Y. Z. Jiang, M. Tang, L. L. Wu, H. Y. Xie, Z. L. Zhang and D. W. Pang, Anal. Chem., 2019, 91, 1178-1184. 